\title{
PERFIL DE RIESGO DE LAS COOPERATIVAS DE CRÉDITO ESPAÑOLAS: IMPLICACIONES EN EL COSTE DEL SEGURO DE DEPÓSITO
}

\author{
POR \\ Pilar GÓMEZ FERNÁNDEZ-AGUADO y \\ Antonio PARTAL UREÑA ${ }^{1}$
}

\section{RESUMEN}

La reforma propuesta por la Comisión Europea en julio de 2010, modificando el procedimiento para fijar las aportaciones que deben realizar las entidades de crédito a los sistemas de garantía de depósitos en función de su nivel de riesgo, supondrá cambios sustanciales en las cuotas que pagan actualmente. Este trabajo analiza el perfil de riesgo de las cooperativas españolas durante el período 2007-2010 y cuantifica su efecto sobre las contribuciones al Fondo de Garantía de Depósitos de Entidades de Crédito (FGDEC) aplicando la metodología desarrollada por la Comisión. El estudio concluye con importantes evidencias sobre la evolución del perfil de riesgo del sector durante un periodo de recesión económica y el impacto de esta reforma, valorado a través del número de entidades que aumentarían su aportación y las variaciones en el coste del seguro respecto al sistema vigente.

Palabras claves: Estabilidad financiera, Sistemas de garantía de depósitos, financiación ajustada al riesgo, disciplina de mercado, banca cooperativa.

Claves Econlit: P130, G280, G210, G320, G010

\footnotetext{
${ }^{1}$ Dra. Pilar Gómez Fernández-Aguado, Profesora contratada doctora Departamento de Economía Financiera y Contabilidad, Universidad de Jaén. Correo electrónico: pigomez@ujaen.es

Dr. Antonio Partal Ureña, Profesor Titular de Universidad, Departamento de Economía Financiera y Contabilidad, Universidad de Jaén. Correo electrónico: apartal@ujaen.es
}

REVESCO N 109 - MONOGRÁFICO: La financiación complementaria y la respuesta de la economía social: la situación del "des-crédito" bajo la crisis financiera -

ISSN: 1885-8031 - www.ucm.es/info/revesco

http://dx.doi.org/10.5209/rev_REVE.2012.v109.40654

Fecha de recepción: 28/03/2012

Fecha de aceptación: 04/05/2012 


\title{
RISK PROFILE OF SPANISH CREDIT COOPERATIVES: IMPLICATIONS FOR THE COST OF DEPOSIT INSURANCE
}

\begin{abstract}
The reform proposed by the European Commission in July 2010, modifying the procedure for determining the contributions which the banks must make to the deposit guarantee systems according to their level of risk will imply substantial changes in the fees currently paid. This paper analyzes the risk profile of Spanish cooperatives during 2007-2010 and quantifies their effect on contributions to the Deposit Guarantee Fund for Credit Institutions (FGDEC) using the methodology proposed by the Commission. The study concludes with important evidence about the evolution of the risk profile of the sector in a period of economic recession and the impact of this reform, measured by means of the number of entities that would increase their input and variations in the cost of insurance in relation to the current system.
\end{abstract}

Keywords: Financial stability, Deposit guarantee systems, Risk-adjusted funding, market discipline, cooperative banking.

\section{INTRODUCCIÓN}

El papel fundamental atribuido a los sistemas de garantía de depósitos (SGD) es estabilizar el sistema financiero en caso de quiebra bancaria, avalando a los depositantes el acceso a sus fondos. Además, de llevar a cabo medidas que permitan un proceso ordenado de gestión en caso de insolvencias bancarias, contribuyendo a limitar el coste de la crisis. La reciente crisis financiera ha demostrado que los SGD europeos no están financiados adecuadamente, siendo el Estado ${ }^{2}$, y finalmente los contribuyentes, quienes asumen las obligaciones potencialmente atribuidas al seguro de depósitos, evidenciando la necesidad de

\footnotetext{
${ }^{2}$ Los trabajos de Hoggarth, Ridhill y Sinclair (2004) y Blundell-Wignall, Wehinger y Slovik, (2009) sobre las resoluciones políticas adoptadas en las crisis bancarias internacionales, evidencian que la participación gubernamental ha sido una característica importante en los procesos de resolución de crisis sistémicas, a través de apoyo a la liquidez por los bancos centrales y la concesión de importantes garantías gubernamentales, materializándose pocas veces la liquidación del banco, y consecuentemente los acreedores rara vez han sufrido pérdidas.
}

REVESCO No 109 - MONOGRÁFICO: La financiación complementaria y la respuesta de la economía social: la situación del "des-crédito" bajo la crisis financiera -

ISSN: 1885-8031 - www.ucm.es/info/revesco 
acometer reformas que garanticen su eficacia. En esta línea, los Principios Básicos para la implantación de seguros de depósitos efectivos, elaborados por el Comité de Supervisión Bancaria de Basilea y la Asociación Internacional de Aseguradores de Depósitos (BCBS/IADI, 2009 y 2010), junto con la propuesta de reforma de la Directiva de los SGD europeos actualmente en desarrollo por la Comisión Europea $(2010)^{3}$, definen los sistemas de garantía del futuro, centradas todas ellas en reformar el sistema de financiación.

La Comisión Europea (2010) considera necesario para garantizar la confianza y credibilidad en los SGD disponer de recursos financieros proporcionales a sus obligaciones potenciales. Estableciendo un nivel objetivo de fondos del 1,5\% de los depósitos admisibles en un período de transición de 10 años. Al mismo tiempo, para avalar la equidad del sistema de financiación, establece que las contribuciones realizadas por las entidades adheridas sean proporcionales al riesgo asumido. Aquellas instituciones con mayor volumen de riesgo, con una probabilidad de quiebra mayor, deben aportar más, y no una cuota independiente del riesgo, que penaliza a las entidades mejor gestionadas. La incorporación de medidas de riesgo en la financiación de los SGD permitirá evaluar mejor las necesidades de patrimonio y, contribuirá a una mayor disciplina de mercado.

Este proceso de adaptación ha supuesto la aprobación del Real Decreto Ley 16/2011, de 14 de octubre, por el que se crea el Fondo de Garantía de Depósitos de Entidades de Crédito (FGDEC) ${ }^{4}$ y, el Real Decreto Ley 19/2011, de 2 de diciembre, estableciendo una contribución única al FGDEC del 2 por mil para todas las entidades. Resoluciones criticadas por la Unión Nacional de Cooperativas de Crédito (UNACC, 2011/2012) al suponer una penalización al modelo de banca tranquila y minorista, y resultar ajenas al estándar europeo, según el cual un modelo como el de las cooperativas de crédito, en la escala más baja de riesgo, conllevaría aportaciones muy inferiores a las exigidas. Además, el notable incremento de aportaciones respecto a las actuales, ocasionará una merma considerable en los resultados de estas entidades.

\footnotetext{
3 Propuesta de Directiva del Parlamento Europeo y del Consejo relativa a los sistemas de garantía de depósitos (refundición), COM (2010)368 final. La finalización del desarrollo normativo y entrada en vigor está prevista para diciembre de 2012:

${ }^{4}$ Con la entrada en vigor del RDL 16/2011, se declara la disolución de los tres Fondos de Garantía de Depósitos existentes hasta el momento (Fondo de Garantía de Depósitos en Establecimientos Bancarios, Fondo de Garantía de Depósitos en Cajas de Ahorro y Fondos de Garantía de Depósitos en Cooperativas de Crédito. El nuevo Fondo tienen por objeto, como venía siendo hasta ahora, garantizar los depósitos en entidades de crédito y realizar actuaciones que refuercen la solvencia y el funcionamiento de las entidades de crédito en dificultades.
}

REVESCO No 109 - MONOGRÁFICO: La financiación complementaria y la respuesta de la economía social: la situación del "des-crédito" bajo la crisis financiera -

ISSN: 1885-8031 - www.ucm.es/info/revesco 
El informe presentado por la Comisión Europea de Asuntos Económicos y Monetarios (2011) sobre la propuesta de Directiva relativa a los SGD subraya la necesidad de considerar los diferentes modelos de negocio desarrollados por las entidades de crédito para establecer su perfil de riesgo. Teniendo en cuenta que la naturaleza de los riesgos que pueden aceptar las entidades asociadas al SGD pueden variar según las circunstancias del mercado o las actividades comerciales desarrolladas, un procedimiento único para el cálculo de las aportaciones no sería razonable, restando equidad al sistema y distorsionando el incentivo de operar con modelos de negocio menos arriesgados. Consideración aprobada por el Parlamento Europeo $(2012)^{5}$, estableciendo que las contribuciones deben ser proporcionales al riesgo comercial de la entidad y considerar el perfil de riesgo de los distintos modelos empresariales.

Las graves consecuencias de la crisis financiera en España han obligado a tomar importantes medidas regulatorias que están conformando un nuevo panorama bancario, donde la mayor aversión al riesgo por parte de las entidades puede condicionar la predisposición a financiar actividades empresariales y determinadas zonas consideradas de mayor riesgo. Aspectos que resaltan aún más la importancia de la banca cooperativa para evitar el aislamiento de determinados territorios como tradicionalmente han venido realizando (Melián, Sanchis y Soler, 2010; Cortes y Belmonte, 2010), y la necesidad, de realizar más investigaciones en el sector, poco estudiado en España (Belmonte, 2012). Un paso en esa dirección es examinar el impacto de las reformas regulatorias en curso.

La finalidad de este trabajo es analizar el perfil de riesgo de las cooperativas de crédito españolas durante el período 2007-2010 y valorar su efecto sobre las contribuciones al Fondo de Garantía de Depósitos de Entidades de Crédito (FGDEC), aplicando el modelo de contribuciones ajustadas al riesgo propuesto por la Comisión Europea (2010). Es necesario e importante precisar, por el período de recesión económica al que está referenciado el estudio, que éste puede ser considerado como un ejercicio de estrés (stress testing) al realizarse en un escenario extremo.

El documento está estructurado como sigue: Sección 2 examina la reforma de los sistemas de financiación europeos. La sección 3 desarrolla el modelo de contribuciones

\footnotetext{
5 Enmienda 107 de la Resolución legislativa del Parlamento Europeo, de 16 de febrero de 2012, sobre la propuesta de Directiva del Parlamento Europeo y del Consejo, relativa a los sistemas de garantía de depósitos (refundición).
}

REVESCO No 109 - MONOGRÁFICO: La financiación complementaria y la respuesta de la economía social: la situación del "des-crédito" bajo la crisis financiera -

ISSN: 1885-8031 - www.ucm.es/info/revesco 
ajustadas al riesgo presentado por la Comisión Europea (2010). La sección 4 analiza el perfil de riesgo y determina el impacto de la aplicación del sistema de aportaciones en base al riesgo en relación al modelo de contribución vigente. Sección 5 concluye.

\section{REFORMA DE LOS SISTEMAS DE FINACIACIÓN EN LOS SGD EUROPEOS}

Actualmente, coexisten en el ámbito europeo mecanismos de financiación ex-ante, expost y mixtos. La financiación ex-ante requiere la constitución anticipada y el mantenimiento de un fondo a partir de las aportaciones realizadas para hacer frente a los pagos derivados del seguro ante la quiebra de una entidad adherida. En los sistemas de financiación ex-post, los fondos son aportados por las entidades miembros cuando se produce la quiebra ${ }^{6}$. Los sistemas de financiación híbridos o mixtos son una combinación de los anteriores, el fondo se nutre de aportaciones periódicas y extraordinarias en caso de contingencia. La tabla $\mathrm{n}^{\mathbf{0}} 1$ muestra las principales características de los sistemas de financiación de los SGD en la UE.

Tabla 1. Características de los sistemas de financiación de los SGD en UE.

\begin{tabular}{|l|l|l|l|l|}
\hline \multirow{2}{*}{ PAÍ́S } & SGD & Tipo de financiación & $\begin{array}{l}\text { Contribución } \\
\text { pública }\end{array}$ & $\begin{array}{l}\text { Contribución } \\
\text { ajustada al } \\
\text { riesgo }\end{array}$ \\
\hline \multirow{2}{*}{ Alemania } & $\begin{array}{l}\text { The Deposit Protection Fund of The German } \\
\text { Private Commercial Banks }\end{array}$ & Ex-ante & No & No \\
\cline { 2 - 5 } & $\begin{array}{l}\text { Deposit Protection Scheme of The National } \\
\text { Austria }\end{array}$ & $\begin{array}{l}\text { Mixta } \\
\text { Commercial Banks }\end{array}$ & No & Si \\
\hline \multirow{2}{*}{ Bélgica } & $\begin{array}{l}\text { Deposit and Financial Instrument Protection Fund } \\
\text { Deprative Banks }\end{array}$ & Ex-ante & No & Si \\
\hline
\end{tabular}

\footnotetext{
${ }^{6}$ Bernet y Walter (2009) analizan las ventajas y limitaciones de los sistemas ex-ante y ex-post, concluyendo en la supremacía del primero. Roy (2000) valora los diferentes sistemas en relación a su grado de eficacia y eficiencia, destacando que el sistema ex-ante es más eficaz y el sistema ex-post más eficiente. En esta línea, el trabajo de Rotte (2011) revela que el sistema ex-ante posee más ventajas que el ex-post a excepción de la transparencia y eficiencia.
}

REVESCO N 109 - MONOGRÁFICO: La financiación complementaria y la respuesta de la economía social: la situación del "des-crédito" bajo la crisis financiera -

ISSN: 1885-8031 - www.ucm.es/info/revesco 


\begin{tabular}{|c|c|c|c|c|}
\hline PAÍS & SGD & Tipo de financiación & $\begin{array}{l}\text { Contribución } \\
\text { pública }\end{array}$ & $\begin{array}{l}\text { Contribución } \\
\text { ajustada al } \\
\text { riesgo }\end{array}$ \\
\hline Bulgaria & Bulgarian Deposit Insurance Fund & Ex-ante & $\mathrm{Si}$ & No \\
\hline Chipre & Deposit Protection Scheme & Mixta & No & No \\
\hline Dinamarca & The Guarantee Fund for Depositors Investors & Mixta & No & No \\
\hline Eslovaquia & Deposit Protection Fund & Ex-ante & No & No \\
\hline Eslovenia & Deposit Gurantee Scheme & Ex-post & No & $\mathrm{Si}$ \\
\hline España & Deposit Guarantee Fund for Banking Institutions & Ex-ante & No & No \\
\hline Estonia & Deposit Guarantee Sectoral Fund & Ex-ante & No & No \\
\hline Finlandia & Deposit Guarantee Fund & Ex-ante & $\mathrm{Si}$ & $\mathrm{Si}$ \\
\hline Francia & Deposit Guarantee Fund & Mixta & No & $\mathrm{Si}$ \\
\hline Grecia & Hellenic Deposit Gurantee Fund & Ex-ante & No & No \\
\hline Hungría & National Deposit Insurance Fund of Hungary & Ex-ante & No & No \\
\hline Irlanda & The Irish Deposit Protection Scheme & Ex-ante & No & No \\
\hline \multirow{2}{*}{ Italia } & Interbank Deposit Protection Fund & Ex-post & No & $\mathrm{Si}$ \\
\hline & $\begin{array}{l}\text { Deposit Guarantee Fund of Cooperative Credit } \\
\text { Banks }\end{array}$ & Ex-post & No & No \\
\hline Letonia & State Company Deposit and Investment Insurance & Ex-ante & $\mathrm{Si}$ & No \\
\hline Lituania & Deposit and Investment Insurance & Ex-ante & $\mathrm{Si}$ & No \\
\hline Luxemburgo & Deposit Gurantee System & Ex-post & No & No \\
\hline Malta & Depositor Compensation Scheme & Mixta & No & No \\
\hline
\end{tabular}

REVESCO No 109 - MONOGRÁFICO: La financiación complementaria y la respuesta de la economía social: la situación del "des-crédito" bajo la crisis financiera -

ISSN: 1885-8031 - www.ucm.es/info/revesco 


\begin{tabular}{|l|l|l|l|l|}
\hline PAí́ & SGD & Tipo de financiación & $\begin{array}{l}\text { Contribución } \\
\text { pública }\end{array}$ & $\begin{array}{l}\text { Contribución } \\
\text { ajustada al } \\
\text { riesgo }\end{array}$ \\
\hline Países Bajos & $\begin{array}{l}\text { Collective Guarantee Scheme of credit institutions } \\
\text { for repayable funds and portfolio investments }\end{array}$ & Ex-post & No & No \\
\hline Polonia & Bank Guarantee Fund & Mixta & Si & No \\
\hline Portugal & Deposit Guarantee Fund & Ex-ante & No & Si \\
\hline Reino Unido & Financial Services Compensation Scheme & Mixta & No & No \\
\hline Republica & Deposit Insurance Fund & Ex-ante & No & No \\
\hline Rumania & Deposit Guarantee Fund & Mixta & No & Si \\
\hline Suecia & Deposit Guarantee Board & Ex-ante & No & Si \\
\hline
\end{tabular}

Fuente: European Forum of Deposit Insurers (2006) y European Commission (2008).

La Comisión Europea (2006) presentó a consulta la posibilidad de homogenizar el marco existente con la implantación única del sistema ex-ante. Los países partidarios alegaban que las diferencias dificultan la capacidad de actuación transfronteriza ante situaciones de crisis que menoscaban la competencia, proporcionando ventajas indebidas a los bancos que operan en el marco de sistemas con menores costes, y un obstáculo para los que desean concentrar sus actividades al amparo del Estatuto de la Sociedad Europea. Mientras, los detractores de la propuesta, argumentaban los elevados costes que implicaría la transición de sistemas ex-post a sistemas ex-ante, cuando el sistema funciona relativamente bien y es adecuado a efectos de supervisión, además, los problemas de concentración transfronteriza sólo afectarían a un número limitado de entidades, no justificándose la introducción de cambios.

A pesar de las opiniones en contra, la Comisión considera fundamental intensificar la integración financiera transfronteriza en Europa, ante el riesgo de un elevado coste a largo plazo por no alcanzar una armonización suficiente en las redes de seguridad, dificultando el funcionamiento adecuado en situaciones de crisis transfronterizas. Los costes en términos

REVESCO Nº 109 - MONOGRÁFICO: La financiación complementaria y la respuesta de la economía social: la situación del "des-crédito" bajo la crisis financiera -

ISSN: 1885-8031 - www.ucm.es/info/revesco 
económicos y el deterioro de la confianza en el mercado financiero único podrían, en última instancia, resultar mucho mayores que la inversión necesaria para el funcionamiento satisfactorio de la red de seguridad panaeuropea. El informe Scenario Analysis: Estimating the effects of changing the funding mechanisms of EU Deposit Guarantee Schemes realizado por la Comisión (2007), estimó el coste de armonizar los mecanismos de financiación entre 2.500 y 4.300 millones de euros en un plazo de 10 años para los países que actualmente se financian ex-post.

La Comisión, conforme a su plan de mejora del marco legislativo de los SGD, pretende ir más allá de la simple implantación de sistemas de financiación ex-ante, estableciendo aportaciones en función del perfil de riesgo de la entidad. Si bien, la Directiva 94/19/CE autoriza la introducción de elementos basados en el riesgo ${ }^{7}$, una armonización exigiría una importante y compleja modificación, no viable a corto plazo, siendo necesario analizar la situación actual y el impacto de la reforma. En este sentido, presentó en junio de 2008 el informe Risk-based contributions in EU Deposit Guarantee Schemes: current practices, detallando distintos modelos de contribución en base al riesgo aplicados por Alemania, Francia, Italia, Portugal, Finlandia y Suecia, y posteriormente el informe Possible models for risk-based contributions to EU Deposit Guarantee Sechemes en junio de 2009, con tres alternativas para el cálculo de las contribuciones: Modelo de indicador único ${ }^{8}$, Modelo de indicadores múltiples ${ }^{9}$ y Modelo de riesgo de impago ${ }^{10}$. Los dos primeros utilizan

\footnotetext{
${ }^{7}$ En los SGD europeos que emplean modelos de financiación ajustados al riesgo (Alemania, Francia, Italia, Portugal, Finlandia y Suecia), la contribución anual de las entidades adheridas generalmente se establece en función de la base de cotización o total de depósitos elegibles o cubiertos y un coeficiente representativo del perfil de riesgo de la entidad. El perfil de riesgo es evaluado a través de diferentes indicadores financieros que determinan el grado de solvencia, exposición al riesgo y rentabilidad de la entidad. En función de la evaluación obtenida, las entidades son clasificadas en categorías de riesgo y asociadas a un coeficiente de ponderación de riesgo que determinará la contribución a realizar.

${ }^{8}$ El modelo de indicador único utiliza para establecer la diferenciación del riesgo y consecuentemente las contribuciones a realizar al seguro, el ratio de capital propuesto por Basilea II. Diferentes estudios en el ámbito académico corroboran la adecuación de utilizar la ratio de capital o algunos de los métodos establecidos en Basilea II para medir el riesgo a fin de establecer un sistema de primas variables objetivo y consistente (Sironi y Zazzara, 2004; Lee, 2008; Gilman, 2009, entre otros).

${ }^{9}$ En el modelo de indicadores múltiples, el coeficiente de ajuste estaría determinado por cuatro indicadores correspondientes a las áreas que comúnmente son utilizadas para evaluar la solidez financiera de un banco: adecuación de capital, calidad de activos, rentabilidad y liquidez. Actualmente, el FEDIC (Federal Deposit Insurance Corporation) emplea un sistema de indicador múltiple basado en sistema de calificación CAMELS.

${ }^{10}$ El modelo de riesgo de impago, está basado en la teoría de valoración de opciones propuesta por Merton (1977), estableciendo una analogía entre el seguro de depósitos y una opción de venta para establecer la probabilidad de impago. El contrato de garantía de depósitos se considera una emisión por parte del sistema de garantía de un derecho de venta a favor de los depositantes sobre los activos bancarios, a un precio de ejercicio igual al valor de los depósitos más los intereses. Esta línea de investigación iniciada hace varios años, ha vuelto a
}

REVESCO No 109 - MONOGRÁFICO: La financiación complementaria y la respuesta de

la economía social: la situación del "des-crédito" bajo la crisis financiera -

ISSN: 1885-8031 - www.ucm.es/info/revesco 
información contable y el tercero además, información de mercado. Para concluir en julio de 2010 con la propuesta legislativa que modificará la Directiva 94/19/CE, desarrollando un modelo específico para el cálculo de las contribuciones a los SGD en función del riesgo respaldado por los estudios e informes previos, y que refleja la tendencia adoptada en varios Estados miembros. Pretende además de incentivar una gestión de riesgos sensata, ser un disuasivo para asumirlos, estableciendo una importante diferenciación en las contribuciones de las entidades con menor o mayor riesgo.

\section{MODELO DE CONTRIBUCIONES AJUSTADAS AL RIESGO PARA LOS SGD EUROPEOS}

La Comisión Europea (2009) consideró que el modelo de contribuciones ajustadas al riesgo debía reunir los siguientes requisitos para ser eficiente:

- Preciso: Cuantificar el riesgo específico y sistémico con la mayor exactitud posible y establecer una diferenciación significativa de las categorías de riesgo.

- Simple: Comprensible por los miembros del SGD, es decir, no debe estar relacionado con fórmulas complejas.

- Razonable: En términos de cantidad de información necesaria (recopilación de datos).

- Sostenible: No debe causar la insolvencia de los miembros, es decir, los costes derivados de la aplicación del modelo deben ser sostenibles.

- Flexible: Ajustable a diferentes países, es decir, economías diferentes, mercados financieros y/o instituciones diferentes.

- Abierto y transparente: Los miembros deben conocer por qué son asignados a una determinada categoría de riesgo y poder verificarlo.

- Equitativo e imparcial: El modelo debe ser justo, es decir, los bancos con características similares recibirán el mismo tratamiento.

cobrar importancia de nuevo con los trabajos de Madan y Pennacchi, 2003; Campos, et. al., 2007; Hwang, et. al., 2009, entre otros.

REVESCO N 109 - MONOGRÁFICO: La financiación complementaria y la respuesta de la economía social: la situación del "des-crédito" bajo la crisis financiera -

ISSN: 1885-8031 - www.ucm.es/info/revesco 
El modelo propuesto por la Comisión Europea (2010), define a priori el nivel objetivo de fondos o importe total de contribuciones a recaudar por el SGD, que posteriormente será repartido entre las entidades adheridas según el perfil de riesgo individual.

La forma de proceder sería, una vez establecida la cantidad total de contribuciones a captar (TC), determinar la aportación de cada miembro $\left(\mathrm{C}_{\mathrm{i}}\right)$ en función de la cuota de riesgo de la entidad, medido a través del coeficiente $\left(\mathrm{RS}_{\mathrm{i}}\right)$ :

$$
\mathrm{C}_{\mathrm{i}}=\mathrm{TC} \cdot \mathrm{RS}_{\mathrm{i}}
$$

El coeficiente $\mathrm{RS}_{\mathrm{i}}$, es el resultado del cociente entre la contribución de cada miembro $\left(\mathrm{RA}_{\mathrm{i}}\right)$ y el importe total de las contribuciones al sistema, ambas ponderadas por riesgo:

$$
\mathrm{RS}_{\mathrm{i}}=\frac{\mathrm{RA}_{\mathrm{i}}}{\sum_{\mathrm{j}=1}^{\mathrm{n}} \mathrm{RA}_{\mathrm{j}}}
$$

A su vez, la aportación de cada entidad $\left(\mathrm{RA}_{\mathrm{i}}\right)$, vendrá determinada por el producto del riesgo individual $\left(\beta_{\mathrm{i}}\right)$ y la exposición al riesgo $\left(\mathrm{CB}_{\mathrm{i}}\right)$ definida por los depósitos elegibles o cubiertos que determinan la base de contribución:

$$
\mathrm{RA}_{\mathrm{i}}=\mathrm{CB}_{\mathrm{i}} \cdot \beta_{\mathrm{i}}
$$

El coeficiente de riesgo individual $\left(\beta_{\mathrm{i}}\right)$, obtenido en función de la puntuación compuesta de riesgo $\left(\rho_{\mathrm{i}}\right)$ de cada miembro, puede variar entre un $75 \%$ para entidades con riesgo mínimo y el 200\% para aquellas con riesgo extremo. Penalizando hasta con el doble de aportaciones a las que obtengan una valoración de riesgo por encima de la media, y beneficiando con disminuciones de hasta un $25 \%$ a las que se encuentren por debajo.

La puntuación compuesta de riesgo $\left(\rho_{\mathrm{i}}\right)$ resulta de un conjunto de indicadores que cubren las categorías de riesgos más importantes de las entidades y generalmente utilizados para evaluar la solvencia: adecuación de capital, calidad de los activos, rentabilidad y

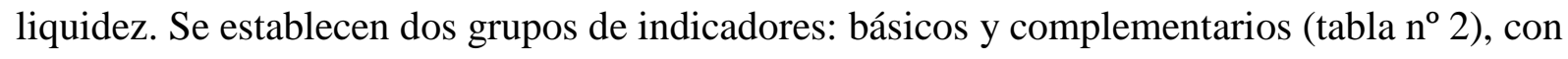
ponderaciones del $75 \%$ y 25\%, respectivamente. Los indicadores básicos serán fijados por la Comisión Europea y aplicados a todos los SGD europeos. Los Estados miembros determinarán los indicadores complementarios aplicables a su jurisdicción, a este efecto la Comisión ofrece algunas orientaciones. 
Tabla 2. Indicadores básicos y complementarios.

\begin{tabular}{|c|c|c|c|c|}
\hline \multirow{2}{*}{$\begin{array}{l}\text { CATEGORÍA } \\
\text { DE RIESGO }\end{array}$} & \multicolumn{2}{|r|}{ INDICADORES BÁSICOS } & \multicolumn{2}{|c|}{ INDICADORES COMPLEMENTARIOS } \\
\hline & Indicador & Medida & Indicador & Medida \\
\hline \multirow[b]{2}{*}{$\begin{array}{l}\text { ADECUACIÓN DE } \\
\text { CAPITAL }\end{array}$} & \multirow{2}{*}{$\begin{array}{l}\text { Ratio de } \\
\text { solvencia } \\
\text { (normativa } \\
\text { BIS) }\end{array}$} & \multirow[b]{2}{*}{$\mathrm{AC} 1=\frac{\text { Fondos Propios }}{\text { Activos Ponderados por riesgo }}$} & Ratio Tier 1 & $\mathrm{AC} 2=\frac{\text { Capital Tier } 1}{\text { Activos Ponderados por riesgo }}$ \\
\hline & & & $\begin{array}{l}\text { Ratio de } \\
\text { Capital }\end{array}$ & $\begin{array}{l}\text { AC3 }=\frac{\text { Capital excedentario }}{\text { Activo Total }} \\
\text { AC4 }=\frac{\text { Capital excedentario }}{\text { Activos ponderados por riesgo }}\end{array}$ \\
\hline \multirow{2}{*}{$\begin{array}{l}\text { CALIDAD DE LOS } \\
\text { ACTIVOS }\end{array}$} & \multirow{2}{*}{$\begin{array}{l}\text { Ratio de } \\
\text { morosidad }\end{array}$} & \multirow{2}{*}{$\mathrm{CA} 1=\frac{\text { Activos dudosos }}{\text { Inversión crediticia }}$} & $\begin{array}{l}\text { Ratio de } \\
\text { activos } \\
\text { ponderados } \\
\text { en función } \\
\text { del riesgo }\end{array}$ & $\mathrm{CA} 2=\frac{\text { Activos ponderados por riesgo }}{\text { Activo total }}$ \\
\hline & & & $\begin{array}{l}\text { Ratio de } \\
\text { provisión }\end{array}$ & $\begin{array}{l}\mathrm{CA} 3=\frac{\text { Provisión para préstamos incobrables }}{\text { Ingresos netos por intereses }} \\
\text { CA4 }=\frac{\text { Provisión para préstamos incobrables }}{\text { Ingresos de explotación }}\end{array}$ \\
\hline \multirow{2}{*}{ RENTABILIDAD } & \multirow{2}{*}{$\begin{array}{l}\text { Ratio de } \\
\text { rentabilidad } \\
\text { del activo }\end{array}$} & \multirow{2}{*}{$\mathrm{R} 1=\frac{\text { Beneficio neto }}{\text { Activo total medio }}$} & $\begin{array}{l}\text { Ratio } \\
\text { costes- } \\
\text { ingresos }\end{array}$ & $\mathrm{R} 2=\frac{\text { Gastos de explotación }}{\text { Ingresos de explotación }}$ \\
\hline & & & $\begin{array}{l}\text { Margen } \\
\text { neto }\end{array}$ & $\mathrm{R} 3=\frac{\text { Beneficio neto }}{\text { Capital Total }}$ \\
\hline LIQUIDEZ & \multicolumn{4}{|c|}{ A determinar por los Estados miembros. } \\
\hline
\end{tabular}

Fuente: European Commission (2010).

Si bien, algunos aspectos metodológicos de la propuesta aún no están completamente especificados (procedimiento para la asignar las puntuaciones por riesgo de los indicadores complementarios y el indicador básico de liquidez), previsiblemente el 31 de diciembre de 2012 como fecha máxima, la Autoridad Bancaria Europea emitirá las directrices necesarias para completarla. No obstante, el planteamiento general adolece de ciertas limitaciones que le restan eficacia, concretamente, no considera el riesgo sistémico en la contribución. Aspecto 
considerado básico por la Comisión (2009), y sin embargo, omitido en el desarrollo posterior, donde sólo reconoce los riesgos individuales de las entidades adheridas.

No considerar ninguna medida que cuantifique el impacto de factores determinantes del riesgo sistémico, como correlaciones entre inversiones de los bancos (Acharya y Yorulmazer, 2008), el gran tamaño de algunas entidades (O’Hara y Shaw, 1990) o la interconexión bancaria (Allen y Gale, 2000; Kahn y Santos, 2008), provoca que bancos con mayor riesgo sistémico sean subvencionados por el resto, distorsionando la equidad del sistema (Penachi, 2006). Cuando es evidente el mayor coste que supone la quiebra de bancos grandes para el seguro de depósitos por las pérdidas que origina su liquidación y el efecto contagio, en consecuencia, la contribución al seguro debe ser mayor a la realizada por los bancos de reducido tamaño (Acharya, Santos y Yorulmazer., 2010). En la práctica, cuantificar el riesgo sistémico puede ser todo un reto, pero los recientes avances en esta línea (Adrian y Brunnermeier, 2008 y Acharya et al., 2010) presentan algunos enfoques a considerar en la revisión de los sistemas de financiación en SGD.

\section{IMPACTO DE UN SISTEMA DE FINANCIACIÓN BASADO EN RIESGO PARA LAS CONTRIBUCIONES DE LAS COOPERATIVAS DEL CREDITO AL FGDEC}

El objetivo es valorar como afectaría a las cooperativas de crédito españolas adheridas al FGDEC la aplicación del modelo de financiación basado en riesgo propuesto por la Comisión Europea (2010). Analizando en primer lugar la evolución del perfil de riesgo entre los años 2007-2010, y posteriormente, cuantificando el impacto comparando las estimaciones de las contribuciones ajustadas al riesgo con las aportaciones vigentes durante el período.

La muestra utilizada la componen 50 cooperativas de crédito adheridas al FGDEC durante el período 2007-10, con una representatividad del 97,3\% respecto al volumen de activo total medio de la población. La composición de la muestra es constante durante todo el período, de esta forma, las variaciones en los resultados no responden a cambios en la composición, sino a la evolución efectiva del perfil de riesgo. La información financiera procede de informes financieros públicos (cuentas anuales, memorias e informes con relevancia prudencial).

REVESCO No 109 - MONOGRÁFICO: La financiación complementaria y la respuesta de la economía social: la situación del "des-crédito" bajo la crisis financiera -

ISSN: 1885-8031 - www.ucm.es/info/revesco 
Los indicadores de riesgo utilizados han sido los básicos propuestos por la Comisión: ratio de solvencia (AC1), ratio de morosidad (CA1) y ratio de rentabilidad del activo (R1); como indicadores complementarios $^{11}$ : ratio de capital (AC3), ratio de provisión (CA3) y ratio de rentabilidad financiera (R3). Respecto a los indicadores de liquidez, al estar su elección sujeta a discrecionalidad del Estado miembro, consideramos para el estudio ratios comúnmente utilizado por el sector para estimar el riesgo de liquidez: ratio de liquidez inmediata ( $\mathrm{L} 1$ = Activos líquidos ${ }^{12} /$ Depósitos de la clientela) como indicador básico y, ratio de liquidez estructural (L2 = Crédito a la clientela/Depósitos de la clientela) como complementario.

La metodología utiliza para determinar el perfil de riesgo y estimar las contribuciones ajustadas al riesgo sigue las directrices marcadas por la Comisión (2010), si bien, al encontrarse en fase de desarrollo, existen aspectos metodológicos pendientes de concretar que requieren considerar algunas hipótesis:

1. Los niveles de riesgo para los indicadores básicos (AC1, CA1 y R1) son definidos con la escala de valores propuesta por la Comisión (Tablas $\mathrm{n}^{\mathrm{o}} 1$ y 2 del anexo I), y para los indicadores complementarios (AC3, CA3, R3, y L2) e indicador básico de liquidez (L1) con los percentiles de la distribución de valores de cada indicador (Tabla $\mathrm{n}^{\mathrm{o}} 3$ del anexo I). Esta consideración implica que los resultados obtenidos con los indicadores AC1, CA1 y R1, muestran la posición de riesgo de las cooperativas de crédito españolas respecto al estándar requerido por la Comisión a las entidades de crédito europeas, y para el resto de indicadores representan la distribución de riesgo en el sector cada año.

2. El perfil de riesgo de cada miembro dependerá de las puntuaciones alcanzada en los indicadores básicos (AC1, CA1, R1 y L1) y complementarios (AC3, CA3, R3, y L2). La ponderación de los indicadores básicos en la puntuación compuesta es un $75 \%$, y los complementarios un $25 \%$. El coeficiente de riesgo ( $\left.\beta_{\mathrm{i}}\right)$ se determina en función de la puntuación compuesta según establece la Comisión (tabla nº 4 del anexo I)

\footnotetext{
${ }^{11}$ La selección de los indicadores complementaros ha estado condicionada por la disponibilidad de información.

${ }^{12}$ Los Activos Líquidos comprenden los siguientes conceptos: Caja y depósitos en bancos centrales, Depósitos en entidades de crédito, Valores representativos de deuda e instrumentos de capital disponibles para la venta.
}

REVESCO N 109 - MONOGRÁFICO: La financiación complementaria y la respuesta de la economía social: la situación del "des-crédito" bajo la crisis financiera -

ISSN: 1885-8031 - www.ucm.es/info/revesco 
3. El importe total de las contribuciones recaudadas (TC), es igual a la suma de las aportaciones realizadas por las entidades de la muestra cada año y posteriormente repartida según la cuota de riesgo de cada miembro, de esta forma se facilita la comparación entre los dos sistemas de financiación.

4. La exposición al riesgo de cada entidad equivale a los depósitos garantizados según la normativa española vigente durante el período (RD 2606/1996, de 20 diciembre).

\subsection{Evolución del perfil de riesgo de las cooperativas españolas}

El sistema de calificación o rating propuesto por la Comisión (2010) diferencia cinco niveles de riesgo (mínimo, bajo, medio, alto y extremo), en función de la puntuación obtenida en cada indicador y de la puntuación compuesta. Las características del sistema, permiten valorar el grado de fortaleza o debilidad que presentan las entidades en cada una de las áreas de evaluación del riesgo (adecuación de capital, calidad del activo, rentabilidad y liquidez), y determinar su perfil global consecuencia de la interacción entre los distintos factores de riesgo.

A continuación se detallan los resultados más relevantes en la evolución del perfil de riesgo de las cooperativas de crédito en el período 2007-10 en cada una de las áreas de evaluación y a nivel global (véase Anexo II).

Adecuación del capital. Los niveles de solvencia se han mantenido ampliamente por encima de los mínimos regulatorios. La fortaleza del coeficiente de solvencia (AC1) sitúa a las cooperativas españolas en una excelente posición respecto a las entidades de crédito europeas, distribuyéndose todas entre niveles de riesgo mínimo o bajo, y una evolución positiva a lo largo del período, con el aumento progresivo de cooperativas calificadas en riesgo mínimo (Gráfico $\mathrm{n}^{\mathrm{o}}$ 1). Igual comportamiento mantiene el nivel de apalancamiento (AC3), presentando un crecimiento sostenido para las distintas categorías de riesgo. Esta favorable evolución de la solvencia se debe fundamentalmente al incremento en los niveles de capital y reservas ${ }^{13}$ registrados a lo largo del periodo.

\footnotetext{
${ }^{13}$ En épocas de crisis, la presión regulatoria ocasiona de forma generalizada un aumento de reservas en las entidades de crédito (Cihak y Shaack, 2010).
}

REVESCO No 109 - MONOGRÁFICO: La financiación complementaria y la respuesta de la economía social: la situación del "des-crédito" bajo la crisis financiera -

ISSN: 1885-8031 - www.ucm.es/info/revesco 
Gráfico 1. Distribución de las categorías de riesgo con el coeficiente de solvencia (AC1)

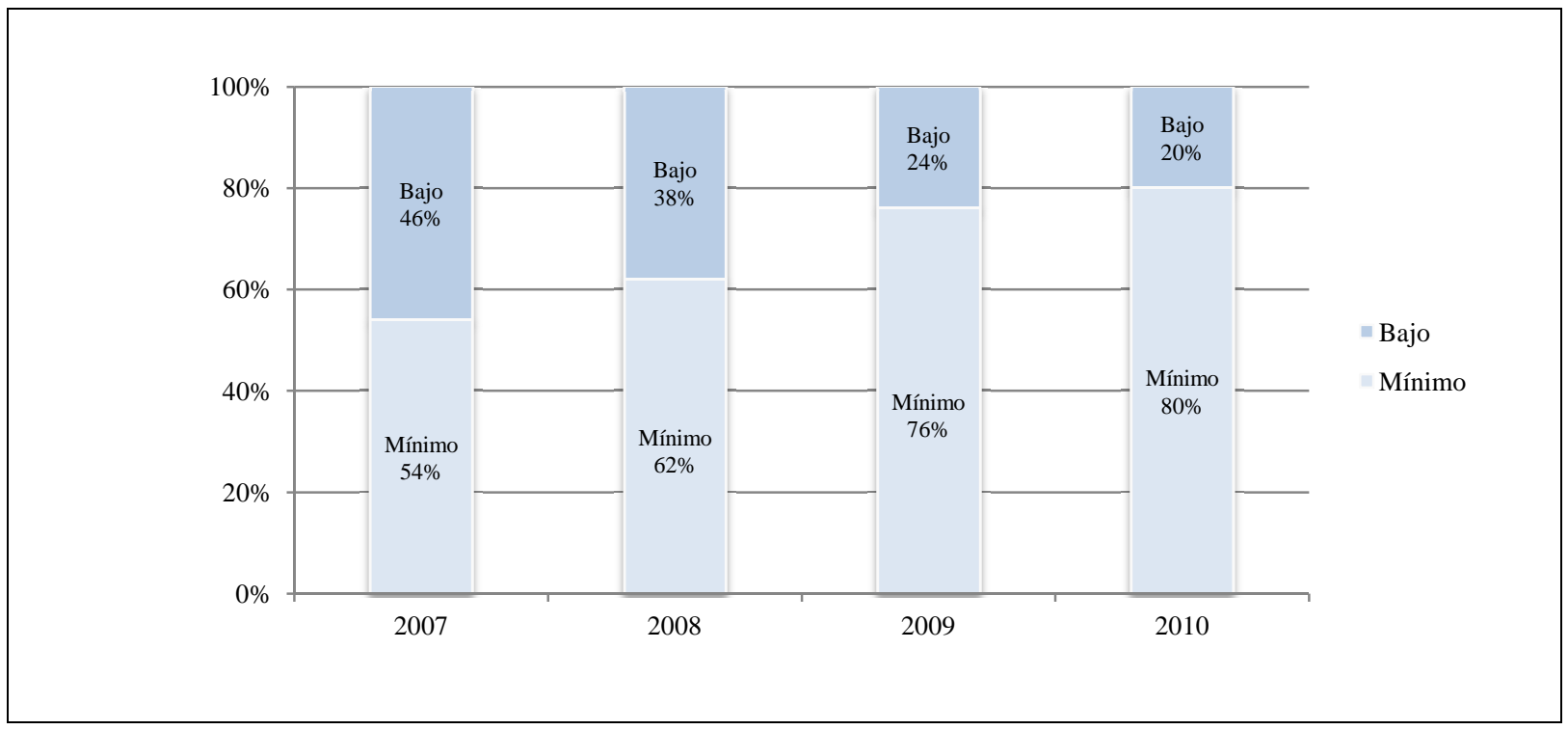

Calidad de los activos. Muestran un rápido e intenso crecimiento de la morosidad (CA1), motivado entre otros aspectos por la expansión crediticia en los años anteriores y la coyuntura económica adversa del período. En 2007, la mayoría de las cooperativas se concentraban en las categorías de riesgo mínimo y bajo respecto al estándar europeo, modificándose sustancialmente el perfil de riesgo en los siguientes años hasta alcanzar en 2010 la mayoría un nivel medio-alto (Gráfico $n^{\circ} 2$ ). En términos generales, el incremento de activos dudosos durante el período ha presionado al alza la ratio de morosidad, si bien, la evolución no es homogénea entre las distintas categorías de riesgo. La ratio presenta una tendencia decreciente en el nivel mínimo, reflejo de la capacidad de selección y gestión de riesgo de las cooperativas incluidas en esta categoría. El crecimiento generalizado de la ratio CA3 en el período 2007-09, manifiesta la incapacidad de las entidades para rentabilizar el riesgo de cartera, causado por un incremento de las provisiones por préstamos incobrables y la reducción del margen de intereses. Tendencia que se invierte en 2010, cuando la relación disminuye de forma generalizada en todas las categorías de riesgo, siendo aún mayor en aquellas con menor riesgo.

REVESCO Nº 109 - MONOGRÁFICO: La financiación complementaria y la respuesta de la economía social: la situación del "des-crédito" bajo la crisis financiera -

ISSN: 1885-8031 - www.ucm.es/info/revesco 
Grafico 2. Distribución de las categorías de riesgo con la ratio de morosidad (CA1)

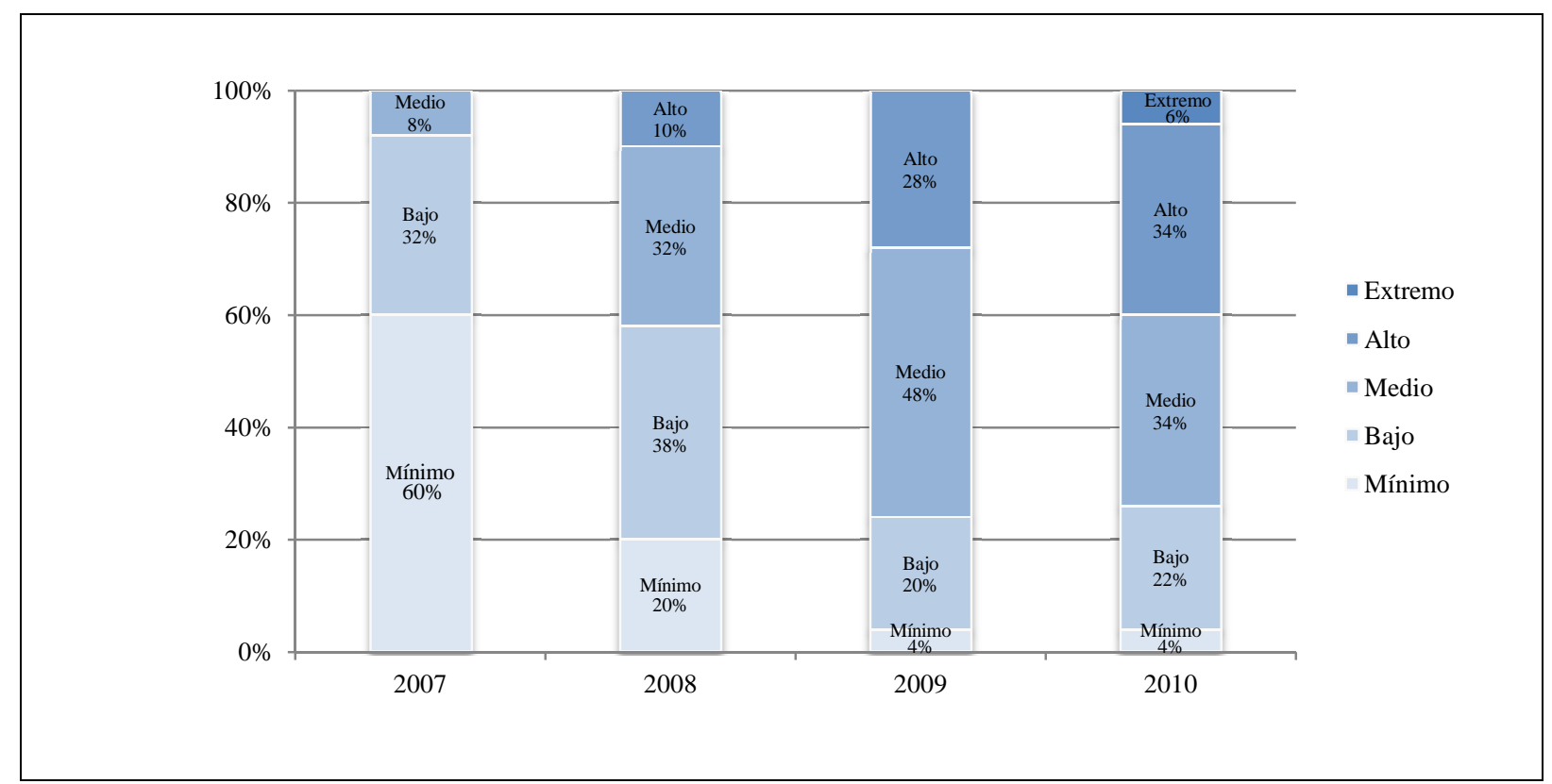

Rentabilidad. Al inicio del período presentaban una sólida posición en términos de rentabilidad económica (R1), la mayoría en niveles de riesgo bajo y medio respecto al estándar europeo (Gráfico $\mathrm{n}^{\mathbf{0}}$ 3). Las dificultades financieras y las consecuencias en la economía real presionaron a la baja los resultados de la actividad, mermando progresivamente la rentabilidad con importantes cambios en la distribución de riesgo (el $74 \%$ de las cooperativas son calificadas con riesgo extremo en 2010 frente al 6\% registrado en 2007). Esta tendencia es homogénea en las categorías de riesgo bajo, medio y alto, siendo significativamente más intensa en el nivel de riesgo extremo. En términos de rentabilidad financiera la tendencia a la baja es de mayor magnitud, consecuencia del efecto conjunto en la caída de resultados y el incremento de la capitalización, más destacado aún en las cooperativas de mayor riesgo.

REVESCO No 109 - MONOGRÁFICO: La financiación complementaria y la respuesta de la economía social: la situación del "des-crédito" bajo la crisis financiera -

ISSN: 1885-8031 - www.ucm.es/info/revesco 
Gráfico 3. Distribución de las categorías de riesgo con la ratio de rentabilidad económica (R1).

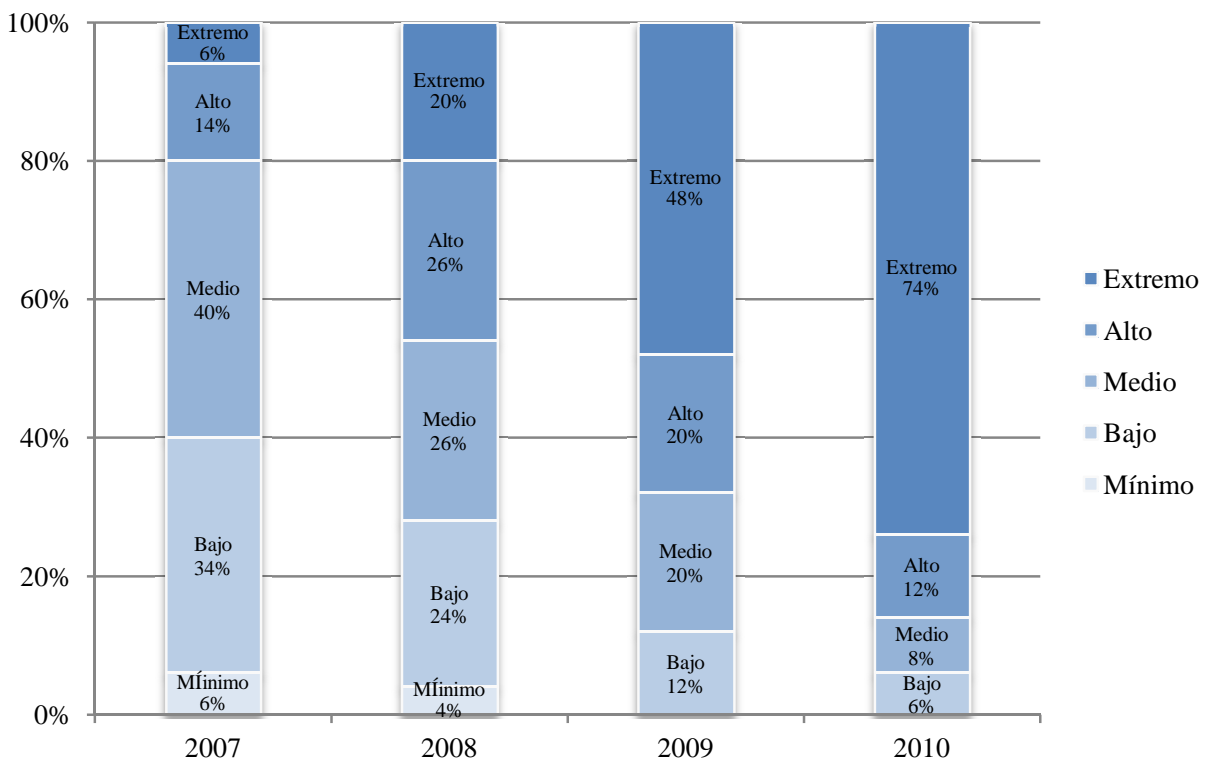

Liquidez. En el año 2008 se constata un descenso generalizado del nivel de liquidez (L1), sin embargo, desde ese momento, se produce una fuerte tendencia alcista, más evidente en las categorías de riesgo bajo y medio. Diferente tendencia presenta el riesgo de liquidez estructural (L2), aumentando en todas las categorías al inicio del período, disminuyendo de forma generalizada en 2009, para volver a crecer en 2010 en los niveles medio, alto y extremo.

Perfil global de riesgo. La interacción de los diferentes indicadores modifica la distribución de riesgo global, definida por solo tres categorías (bajo, medio y alto). Al inicio del periodo las cooperativas agrupadas sobre todo en el nivel de riesgo bajo (75\% en 2007), cambian la tendencia en los años sucesivos hacia un incremento del nivel de riesgo medio (70\% en 2010) a costa del nivel bajo. La evolución del perfil global de riesgo muestra como la fortaleza en términos de solvencia presentada por el sector durante el período, es mitigada por la desfavorable evolución de la rentabilidad y calidad de la cartera crediticia. 
Gráfico 4. Distribución del nivel de riesgo global.

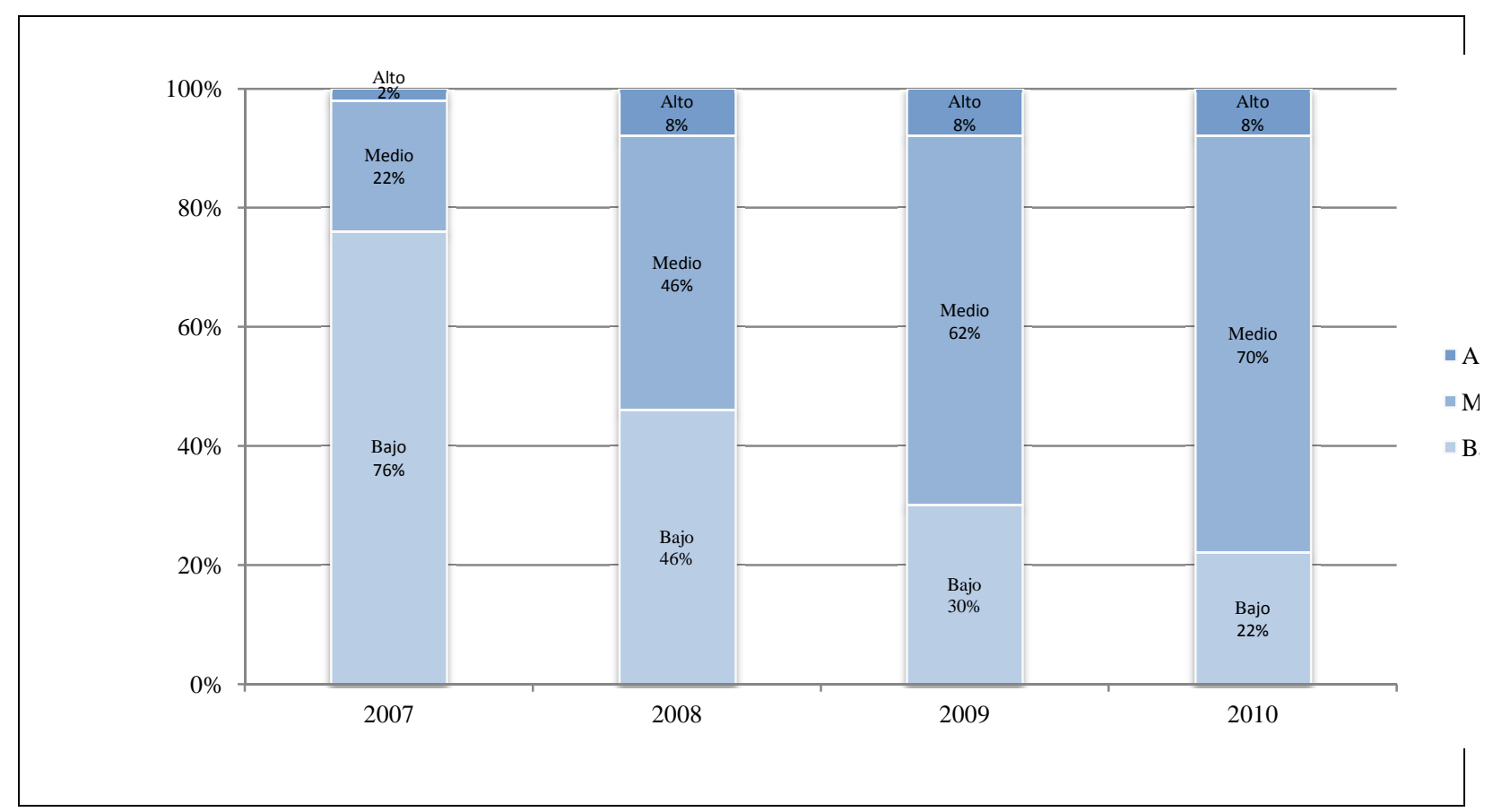

\subsection{Valoración del impacto de las contribuciones ajustadas al riesgo}

Cuantificamos el efecto de la aplicación del sistema de financiación ajustado al riesgo, a partir del porcentaje de cambio relativo entre las contribuciones ajustadas al riesgo y las contribuciones actuales:

$\%$ Cambio en la contribución $=\frac{\text { Contribución ajustada al riesgo }- \text { Contribución actual }}{\text { Contribución actual }} \times 100$

La contribución actual hace referencia a la aportación realizadas por las entidades de la muestra al FGDCC cada año, equivalente al 0,8\% de los depósitos garantizados (RD 2606/1996, de 20 diciembre). La contribución ajustada al riesgo se determina a partir del perfil de riesgo global aplicando la metodología de la Comisión Europea (2010). Los resultados recogidos en la tabla $n^{\circ} 3$, muestran la proporción de entidades que disminuyen o aumentan su contribución con la aplicación del modelo y el rango de variación (aumento porcentual máximo -cambio porcentual máximo positivo- y disminución máxima -cambio porcentual máximo negativo).

REVESCO No 109 - MONOGRÁFICO: La financiación complementaria y la respuesta de la economía social: la situación del "des-crédito" bajo la crisis financiera -

ISSN: 1885-8031 - www.ucm.es/info/revesco 
Tabla 3. Resultados del Modelo de contribuciones ajustadas al riesgo.

\begin{tabular}{|l|c|c|c|c|}
\cline { 2 - 5 } \multicolumn{1}{c|}{} & $\mathbf{2 0 0 7}$ & $\mathbf{2 0 0 8}$ & $\mathbf{2 0 0 9}$ & $\mathbf{2 0 1 0}$ \\
\hline \%Entidades que aumentan aportación & $24,0 \%$ & $54,0 \%$ & $70,0 \%$ & $78,0 \%$ \\
\hline \% Entidades que disminuyen aportación & $76,0 \%$ & $46,0 \%$ & $30,0 \%$ & $22,0 \%$ \\
\hline Aumento porcentual máximo & $37,27 \%$ & $24,86 \%$ & $22,50 \%$ & $20,09 \%$ \\
\hline Disminución porcentual máxima & $-8,48 \%$ & $-16,76 \%$ & $-38,75 \%$ & $-39,95 \%$ \\
\hline
\end{tabular}

La aplicación del modelo de financiación ajustado al riesgo implicaría variaciones considerables para un buen número de entidades respecto a las aportaciones actuales, de hecho, ya en el año 2007, un 24\% de las cooperativas tendrían que haber incrementado su cuota al Fondo, aumentando de forma notable en los años sucesivos, hasta un $78 \%$ en 2010.

El incremento del nivel de riesgo registrado durante el período queda reflejado en la evolución del número de entidades que aumentarían su aportación. Circunstancia que evidencia la prociclicidad implícita del modelo, es decir, en períodos donde la coyuntura económica es favorable y consecuentemente el riesgo del sector es menor, el número de entidades que aportan más al sistema disminuye, aumentando en épocas de recesión. Los modelos ajustados al riesgo pueden generar un adverso impacto procíclico, provocando un aumento de las aportaciones en momentos de recesión económica que agraven la situación (Blinder y Wescott, 2001; Jarrow, Madan y Unal, 2006) ${ }^{14}$.

Respecto al rango de variación en las contribuciones, el modelo muestra la validez para establecer una diferenciación significativa que contribuye a la equidad del sistema,

\footnotetext{
${ }^{14}$ En este contexto, Pennacchi (2005:435), argumenta que un SGD basado en primas ajustadas al riesgo tiene un impacto procíclico menor que un requerimiento de capital ajustado al riesgo como el propuesto en Basilea II. Para paliar estos efectos, Jarrow et al., (2006:1-2) proponen considerar políticas anticíclicas para la determinación de las contribuciones, de manera que el fondo recaudado en tiempos de bonanza económica sea suficiente para atender las obligaciones que se puedan presentar en momentos de crisis.
}

REVESCO No 109 - MONOGRÁFICO: La financiación complementaria y la respuesta de la economía social: la situación del "des-crédito" bajo la crisis financiera -

ISSN: $1885-8031$ - www.ucm.es/info/revesco 
favoreciendo a las entidades para que desarrollen una gestión prudente de las contingencias. Efectos a un más potenciado dependiendo de la coyuntura económica, de hecho, en 2007 cuando existe un mayor número de cooperativas con riesgo bajo, la reducción máxima sería del $8,48 \%$, mientras que en 2010, cuando desciende considerablemente el número de entidades en este grupo, la reducción llega al 39.95\%. Igual sucede con el grupo de entidades que aumentarían la aportación, observando como en 2007 el incremento máximo respecto al vigente es un 37,27\%, y en el año 2010 aumenta un 20,09\%.

\section{CONCLUSIONES}

Aspectos claves para afianzar la estabilidad financiera y prevenir escenarios de crisis sistémica son, entre otros, garantizar la seguridad a los depositantes en las entidades de crédito y una gestión ordenada de las insolvencias bancarias, objetivos encomendados a los SGD. Mecanismos que están siendo objeto de una profunda revisión, por evidenciar que adolecen de importantes limitaciones, principalmente, un sistema de financiación que merma su eficacia y provoca una deficiente disciplina de mercado. Motivos que han llevado a las autoridades europeas e internacionales a buscar medidas que eviten esta arbitrariedad.

La utilización de medidas de riesgo permite evaluar mejor las necesidades de patrimonio y niveles de solvencia necesarios para los SGD y conseguir un sistema de contribuciones más equitativo, favoreciendo la disciplina de mercado. En este contexto, la Comisión Europea (2010) ha planteado un modelo de financiación para los sistemas de garantía basado en contribuciones variables para las entidades en función de su perfil de riesgo. Iniciativa que supone un incentivo regulatorio más para la gestión prudencial de riesgos asociados a la actividad de las entidades de crédito en favor de la estabilidad financiera.

El análisis realizado muestra importantes evidencias sobre el perfil de riesgo de las cooperativas de crédito españolas durante el período 2007-10 y su efecto sobre las contribuciones a realizar al FGDEC. Pese a un entorno económico adverso, el sector ha reforzado su solvencia, manteniendo niveles de capital por encima del mínimo requerido con una posición favorable respecto al estándar establecido por la Comisión. Por el contrario, en términos de rentabilidad y calidad de la cartera crediticia ha sido más vulnerable ante los cambios económicos. Ambos efectos determinan la evolución del riesgo global en el sector,

REVESCO No 109 - MONOGRÁFICO: La financiación complementaria y la respuesta de la economía social: la situación del "des-crédito" bajo la crisis financiera -

ISSN: 1885-8031 - www.ucm.es/info/revesco 
caracterizado al inicio del período por un perfil de riesgo bajo y finalizando con un perfil de riesgo medio. El incremento del nivel de riesgo registrado durante el período tiene un efecto directo sobre el número de entidades que aumentaría su aportación al FGDEC y en el rango de variación que experimentarían las contribuciones respecto a las realizadas en el periodo. Aspectos que si bien corroboran la adecuación de modelo para establecer un sistema de financiación más equitativo, pueden ocasionar cierta prociclicidad en la financiación del Fondo, teniendo que realizar aportaciones más elevadas durante periodos de recesión y reduciéndose las exigencias cuando la coyuntura económica es más favorable. Sin olvidar, además, que el planteamiento inicial de la Comisión adolece de un fuerte limitación al reconocer solo los riesgos individuales de las entidades, obviando factores de riesgo sistémico que deberían ser considerados para mejorar la equidad del sistema. Aspecto que de ser considerado, beneficiaría a las entidades con bajo nivel de riesgo sistémico, como son las cooperativas de crédito.

\section{BIBLIOGRAFÍA}

ACHARYA, V. V. y YORULMAZER, T. Cash-in-the-Market Pricing and Optimal Resolution of Bank Failures. Review of Financial Studies, n²1, vol. 6, 2008, p. 2705-42.

ACHARYA, V. V., SANTOS, J. A. C. y YORULMAZAR, T. Systemic Risk and Deposit Insurance Premiums. FRBNY Economic Policy Review, August, 2010, p. 89-99.

ADRIAN, T., y. BRUNNERMEIER, M. K. CoVar. Federal Reserve Bank of New York, Staff Reports n³48, September, 2008.

ALLEN, F. y GALE, D. Financial Contagion. Journal of Political Economy, nº 108, vol. 1, 2000, p. 1-33.

AYADI, R. y LASTRA, R.M. Proposal for reforming deposit guarantee schemes in Europe. Journal of Banking Regulation, $\mathrm{n}^{\circ} 11,2010$, p. 210-22.

BASEL COMMITTEE ON BANKING SUPERVISION, INTERNATIONAL ASSOCIATION OF DEPOSIT INSURER (BCBS/IADI). Core Principles for Effective Deposit Insurance System, Bank for International Settlements Publications, June, 2009.

BASEL COMMITTEE ON BANKING SUPERVISION, INTERNATIONAL ASSOCIATION OF DEPOSIT INSURER (BCBS/IADI). Core Principles for Effective

REVESCO Nº 109 - MONOGRÁFICO: La financiación complementaria y la respuesta de la economía social: la situación del "des-crédito" bajo la crisis financiera -

ISSN: 1885-8031 - www.ucm.es/info/revesco 
Deposit Insurance Systems: A proposed methodology for compliance assessment, Bank for International Settlements Publications, November, 2010.

BASEL COMMITTEE ON BANKING SUPERVISION. Basel III: A global regulatory framework for more resilient banks and banking systems, Basel Committee Publications, December, 2010.

BASEL COMMITTEE ON BANKING SUPERVISION. International Convergence of Capital Measurement and Capital Standards: A Revised Framework - Comprehensive Version, Basel Committee Publications, June, 2006.

BELMONTE, L. J. El nuevo modelo de negocio de las cooperativas de crédito españolas. Perspectivas futuras. Papeles de economía española, $\mathrm{n}^{\circ}$ 130, 2011 , p. 244-257.

BERNET, B. y WALTER, S. Design, structure and implementation of a modern deposit insurance scheme, The European Money and Finance Forum, Vienna, 2009.

BLINDER, A. S. y WESCOTT, R. F. Reform of deposit insurance: A report to the FDIC. Federal Deposit Insurance Coporation, Washington DC, 2001. Disponible en: http://www.fdic.gov/deposit/insurance/initiative/reform.html

BLUNDELL-WIGNALL, A., WEHINGER, G. Y SLOVIK, P. The Elephant in the Room: The Need to Deal with What Banks Do. OCDE Journal: Financial Market Trends, $\mathrm{n}^{\mathrm{o}} 2$, 2009, p. 1-27.

CAMPOS, P., YAGÜE, M y CHINCHETRU, I. Un nuevo marco de seguro de depósitos para España. Estabilidad Financiera, $\mathrm{n}^{\circ}$ 12, 2007, p. 93-110.

CARIBONI, J., VANDEN, K., CAMPOLONGO, F. y DE CESARE, M. Deposit protection in the UE: State of play and future prospects. Journal of Baking Regulation, n ${ }^{\circ}$ 9, 2008, p. 82-101.

COMMITTEE ON ECONOMIC AND MONETARY AFFAIRS. Report on the proposal for a directive of the European Parliament and of the Council on Deposit Guarantee Schemes (recast). A7-0225/2011. Brussels, June, 2001.

CORTÉS, F.J. y BELMONTE, L.J. La base social de las cooperativas de crédito. La importancia de la responsabilidad social corporativa. Revista de Estudios Empresariales, $\mathrm{n}^{\mathrm{o}} 2,2010$, p. 35-53.

DEMIRGÜC-KUNT, A. y SERVEN, L. Are all the sacred cows dead? Implications of The Financial Crisis form Macro and Financial Policies. The World Bank Research Observer, vol. $25, \mathrm{n}^{\mathrm{o}} 1,2010$, p. 91-124.

REVESCO No 109 - MONOGRÁFICO: La financiación complementaria y la respuesta de la economía social: la situación del "des-crédito" bajo la crisis financiera -

ISSN: 1885-8031 - www.ucm.es/info/revesco 
EUROPEAN COMMISSION. Communication from the Commission to the European Parliament and the Council concerning the review of Directive 94/19/EC on Deposit Guarantee Schemes. COM(2006) 729 final. Brussels, November, 2006.

EUROPEAN COMMISSION. Possible models for risk-based contributions to EU Deposit Guarantee Sechemes. Report, June, 2009.

EUROPEAN COMMISSION. Proposal for a Directive on Deposit Guarantee Schemes EU of The European Parliament and of the Council. COM(2010)368 final. Brussels, July, 2010.

EUROPEAN COMMISSION. Risk-based contributions in EU Deposit Guarantee Schemes: current practices. Report, Ispra (Italy), June, 2008.

EUROPEAN COMMISSION. Scenario Analysis: Estimating the effects of changing the funding mechanisms of EU Deposit Guarantee Schemes. Report, Ispra (Italy), February, 2007.

EUROPEAN FORUN OF DEPOSIT INSURERS. Deposit Guarantee systems: EFDI'S first report. Interbank Deposit Protection Fund. October, 2006. Disponible en: http://www.efdi.net/documents.asp?Id=5\&Cat=Efdi Publications

FINANCIAL STABILITY FORUM. Report of the Financial Stability Forum on Enhancing Market and Institutional Resilience. April, 2008. Disponible en: www.financialstabilityboard.org/publications/r_0804.pdf.

GILBERT, R. A., MAYER, A. P. y VAUGHAN, M. D. The role of a CAMEL Downgrade Model in Bank Surveillance. The Federal Reserve Bank of St. Louis, Working Paper 2000-021A, 2000.

GILMAN, M. International deposit insurance. Economic Affairs, vol. 29, nº 3, 2009, p. 103104.

HOGGARTH, G., REIDHILL, J. y SINCLAIR, P. On the Resolution of Banking Crises: Theory and Evidence. Bank of England, Working Paper, 229, 2004.

HWANG, D. Y., SHIE, F. S., WANG, K. y LIN, J. C. The pricing of deposit insurance considering bankruptcy cost and closure policies. Journal of Banking and Finance, vol. $33, n^{\circ} 10,2009$, p. 1909-1919.

IOANNIDOU, V. P. y PENAS, M. F. Deposit insurance and bank risk-taking: Evidence from internal loan ratings. Journal of Financial Intermediation, vol. 19, n 1, 2010, p. 95-115.

JARROW, R. A., MADAN, D. B. y UNAL, H. Designing countercyclical and risk based aggregate deposit insurance premium. Federal Deposit Insurance Coporation, Center for

REVESCO No 109 - MONOGRÁFICO: La financiación complementaria y la respuesta de la economía social: la situación del "des-crédito" bajo la crisis financiera -

ISSN: 1885-8031 - www.ucm.es/info/revesco 
Financial Research, Working Paper No WP2007-02, 2006. Disponible en: http://papers.ssrn.com/sol3/papers.cfm?abstract_id=964481

KAHN, C. M., y SANTOS, J. A. C. Liquidity, Payment, and Endogenous Financial Fragility. Federal Reserve Bank of New York, 2008. Disponible en: http://laef.ucsb.edu/laef/pages/conferences/pan08/papers/kahn.pdf

LEE, J.P. Internal model-based capital standard and the cost of deposit insurance. Research in Finance, vol. 24, 2008, p. 7-73.

MADAN, D. y PENNACHI, G. Introduction: Special Issue on Pricing the Risk of Deposit Insurance. Journal of Financial Services Research, vol. 24, nº 2, 2003, p. 89-110.

MELIAN, A., SANCHIS, J.M. y SOLER, F. El Crédito Cooperativo como instrumento financiero para el fomento del emprendimiento en tiempos de crisis. CIRIEC-España, Revista de Economía Pública, Social y Cooperativa, nº 68, 2010, p. 111-139.

MERTON, R. An analytic derivation of the cost of deposit insurance and loan guarantees. An application of modern option pricing theory. Journal of Banking and Finance, $\mathrm{n}^{\circ}$ 1, 1977, p. 3-11.

O'HARA, M. Y SHAW, W. Deposit Insurance and Wealth Effects: The Value of Being 'Too Big to Fail. Journal of Finance, vol. 45, n 5, 1990, p. 1587-1600.

PARTAL UREÑA, A. y GÓMEZ FERNÁNDEZ-AGUADO, P. Los sistemas de garantía de depósitos ante la nueva regulación de capital de Basilea. Cuadernos de Información Económica, nº 168, 2002, p. 104-112.

PENNACCHI, G. G. Deposit Insurance, Bank Regulation, and Financial System Risks. Journal of Monetary Economics, vol. 53, n 1, 2006, p. 1-30.

PENNACCHI, G. G. Risk-based capital standards, deposit insurance, and procyclicality. Journal of Financial Intermediation, Vol. 14, 2005, 432-465.

ROTTE, J.G. Moving from ex post to ex ante -the Dutch experience. EFDI Seminar, Belgrado, 2011. Disponible en http://www.efdi.net/events.asp?type=EFDI.

ROY, J. A preliminary analysis of Deposit Insurance Funding Issues. Financial Stability Forum, Study Group on Deposit Insurance, February, 2000, 1-36.

SIRONI, A. y ZAZZARA, C. Applying credit risk models to deposit insurance pricing: Empirical evidence from the Italian banking system. Journal of International Banking Regulation, vol. 6, nº 1, 2004, p. 10-33.

REVESCO No 109 - MONOGRÁFICO: La financiación complementaria y la respuesta de la economía social: la situación del "des-crédito" bajo la crisis financiera -

ISSN: 1885-8031 - www.ucm.es/info/revesco 
UNIÓN NACIONAL DE COOPERATIVAS DE CRÉDITO (UNACC). Legislación. Revista Banca Cooperativa, $\mathrm{n}^{\mathrm{o}}$ 50, Invierno, 2011/2012.

REVESCO No 109 - MONOGRÁFICO: La financiación complementaria y la respuesta de la economía social: la situación del "des-crédito" bajo la crisis financiera -

ISSN: 1885-8031 - www.ucm.es/info/revesco 


\section{ANEXO I}

Tabla 1. Puntuaciones de riesgo para los Indicadores básicos.

\begin{tabular}{|l|c|c|c|c|c|c|}
\hline $\begin{array}{l}\text { Categoría de } \\
\text { riesgo }\end{array}$ & $\begin{array}{c}\text { Ratio } \\
(\mathbf{x})\end{array}$ & $\mathbf{\rho}^{\mathrm{x}}=\mathbf{1}$ & $\boldsymbol{\rho}^{\mathrm{x}}=\mathbf{2}$ & $\mathbf{\rho}^{\mathrm{x}}=\mathbf{3}$ & $\mathbf{\rho}^{\mathrm{x}}=\mathbf{4}$ & $\mathbf{\rho}^{\mathrm{x}}=\mathbf{5}$ \\
\hline $\begin{array}{l}\text { Adecuación } \\
\text { de capital }\end{array}$ & AC & $\mathrm{x}>12,3 \%$ & $12,3 \% \geq \mathrm{x}>9,6 \%$ & $9,6 \% \geq \mathrm{x}>8,2 \%$ & $8,2 \geq \mathrm{x}>7 \%$ & $\mathrm{x} \leq 7 \%$ \\
\hline $\begin{array}{l}\text { Calidad de los } \\
\text { activos }\end{array}$ & CA & $\mathrm{x} \leq 1 \%$ & $1 \%<\mathrm{x} \leq 2,1 \%$ & $2,1 \%<\mathrm{x} \leq 3,7 \%$ & $3,7 \%<\mathrm{x} \leq 6 \%$ & $\mathrm{x}>6 \%$ \\
\hline Rentabilidad & $\mathrm{R}$ & $\mathrm{x}>12 \%$ & $12 \% \geq \mathrm{x}>0,9 \%$ & $0,9 \% \geq \mathrm{x}>0,7 \%$ & $0,7 \% \geq \mathrm{x}>0,5 \%$ & $\mathrm{x} \leq 0,5 \%$ \\
\hline Liquidez & $\mathrm{L}$ & \multicolumn{5}{|c|}{ A determinar por los Estados miembros. } \\
\hline
\end{tabular}

Fuente: European Commission (2010).

Tabla 2. Niveles de riesgo en función de la puntuación de cada indicador.

\begin{tabular}{|l|c|c|c|c|}
\hline Nivel de riesgo & $\begin{array}{c}\text { Adecuación de } \\
\text { capital }\end{array}$ & $\begin{array}{c}\text { Calidad de los } \\
\text { activos }\end{array}$ & Rentabilidad & Liquidez \\
\hline Mínimo & 1 & 1 & 1 & 1 \\
\hline Bajo & 2 & 2 & 2 & 2 \\
\hline Medio & 3 & 3 & 3 & 3 \\
\hline Alto & 4 & 4 & 4 & 4 \\
\hline Extremo & 5 & 5 & 5 & 5 \\
\hline
\end{tabular}

Fuente: European Commission (2010).

Tabla 3. Puntuaciones de riesgo para los Indicadores complementarios e indicador básico de liquidez.

\begin{tabular}{|c|c|c|c|c|c|c|}
\hline \multirow{2}{*}{$\begin{array}{l}\text { Categoría de } \\
\text { riesgo }\end{array}$} & \multirow{2}{*}{ Ratio } & \multicolumn{5}{|c|}{ Puntuación } \\
\hline & & $\rho^{x}=1$ & $\rho^{x}=2$ & $\rho^{x}=3$ & $\rho^{x}=4$ & $\rho^{x}=5$ \\
\hline $\begin{array}{l}\text { Adecuación de } \\
\text { capital }\end{array}$ & $\mathrm{AC} 3$ & $\begin{array}{l}\text { Percentil } \\
60 \text { a } 100 \\
\end{array}$ & $\begin{array}{c}\text { Percentil } \\
40 \text { a } 60 \\
\end{array}$ & $\begin{array}{c}\text { Percentil } \\
25 \text { a } 40 \\
\end{array}$ & $\begin{array}{c}\text { Percentil } \\
10 \text { a } 25 \\
\end{array}$ & $\begin{array}{c}\text { Percentil } \\
0 \text { a } 10\end{array}$ \\
\hline $\begin{array}{l}\text { Calidad de los } \\
\text { activos }\end{array}$ & CA3 & $\begin{array}{c}\text { Percentil } \\
0 \text { a } 20 \\
\end{array}$ & $\begin{array}{c}\text { Percentil } \\
20 \text { a } 40\end{array}$ & $\begin{array}{c}\text { Percentil } \\
40 \text { a } 60\end{array}$ & $\begin{array}{c}\text { Percentil } \\
60 \text { a } 80 \\
\end{array}$ & $\begin{array}{l}\text { Percentil } \\
80 \text { a } 100\end{array}$ \\
\hline Rentabilidad & R3 & $\begin{array}{c}\text { Percentil } \\
0 \text { a } 20\end{array}$ & $\begin{array}{c}\text { Percentil } \\
20 \text { a } 40\end{array}$ & $\begin{array}{c}\text { Percentil } \\
40 \text { a } 60\end{array}$ & $\begin{array}{c}\text { Percentil } \\
60 \text { a } 80\end{array}$ & $\begin{array}{l}\text { Percentil } \\
80 \text { a } 100\end{array}$ \\
\hline \multirow{2}{*}{ Liquidez } & L1 & $\begin{array}{c}\text { Percentil } 80 \\
\text { a } 100\end{array}$ & $\begin{array}{c}\text { Percentil } \\
60 \text { a } 80 \\
\end{array}$ & $\begin{array}{c}\text { Percentil } \\
40 \text { a } 60\end{array}$ & $\begin{array}{c}\text { Percentil } \\
20 \text { a } 40\end{array}$ & $\begin{array}{c}\text { Percentil } \\
0 \text { a } 20\end{array}$ \\
\hline & $\mathrm{L} 2$ & $\begin{array}{c}\text { Percentil } \\
0 \text { a } 20 \\
\end{array}$ & $\begin{array}{c}\text { Percentil } \\
20 \text { a } 40 \\
\end{array}$ & $\begin{array}{c}\text { Percentil } \\
40 \text { a } 60 \\
\end{array}$ & $\begin{array}{c}\text { Percentil } \\
60 \text { a } 80 \\
\end{array}$ & $\begin{array}{l}\text { Percentil } \\
80 \text { a } 100\end{array}$ \\
\hline
\end{tabular}

Fuente: European Commission (2009).

Tabla 4. Escala del coeficiente de riesgo.

\begin{tabular}{|l|c|c|c|c|c|}
\hline Puntuación compuesta $\left(\boldsymbol{\rho}_{\mathbf{i}}\right)$ & $1<\rho_{\mathrm{i}} \leq 1,5$ & $1,5<\rho_{\mathrm{i}} \leq 2,5$ & $2,5<\rho_{\mathrm{i}} \leq 3,5$ & $3,5<\rho_{\mathrm{i}} \leq 4,5$ & $4,5<\rho_{\mathrm{i}} \leq 5$ \\
\hline Coeficiente de riesgo $\left(\boldsymbol{\beta}_{\mathbf{i}}\right)$ & $75 \%$ & $100 \%$ & $125 \%$ & $150 \%$ & $200 \%$ \\
\hline
\end{tabular}

Fuente: European Commission (2010).

REVESCO No 109 - MONOGRÁFICO: La financiación complementaria y la respuesta de la economía social: la situación del "des-crédito" bajo la crisis financiera -

ISSN: 1885-8031 - www.ucm.es/info/revesco 


\section{ANEXO II}

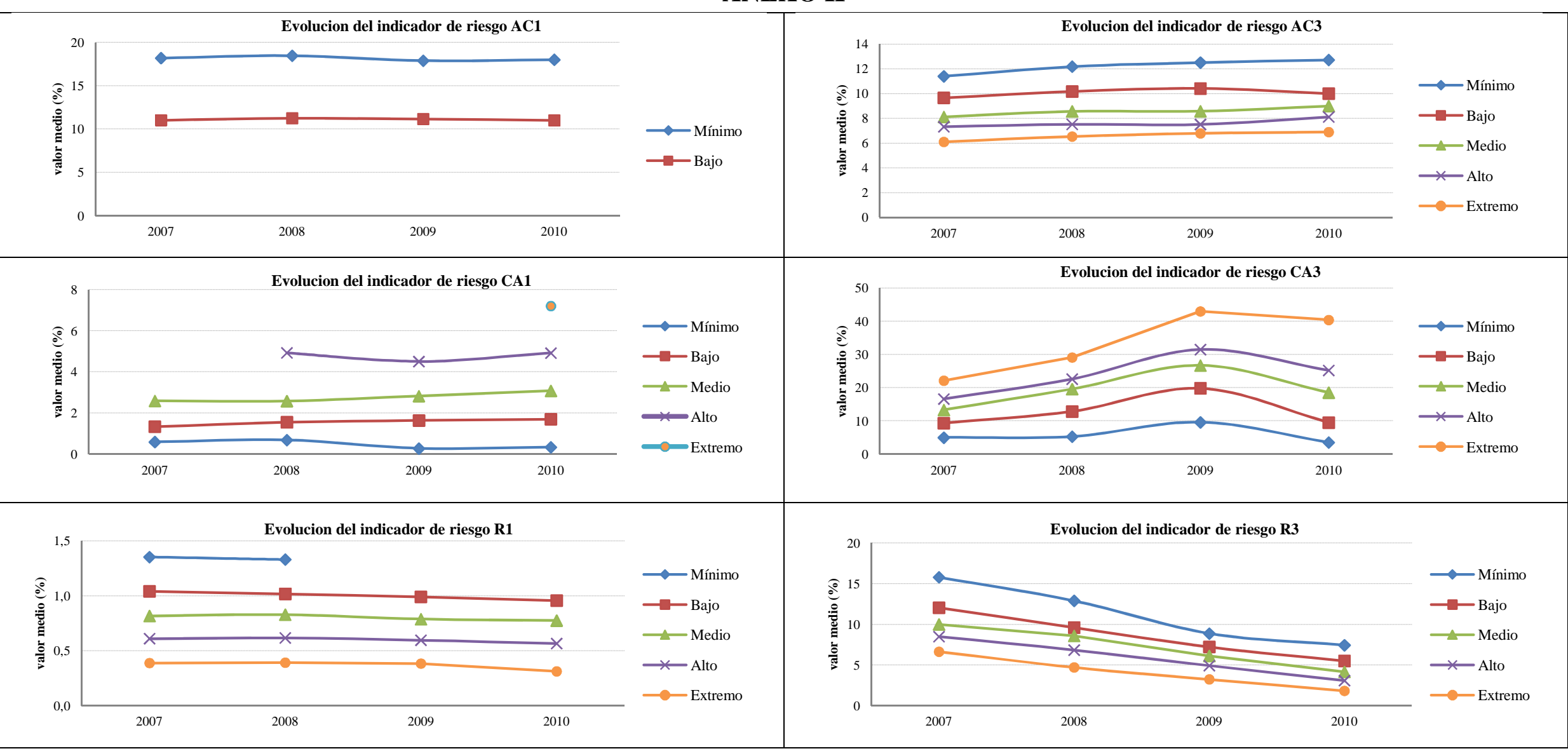

REVESCO No 109 - MONOGRÁFICO: La financiación complementaria y la respuesta de la economía social: la situación del "des-crédito" bajo la crisis financiera -

ISSN: 1885-8031 - www.ucm.es/info/revesco 


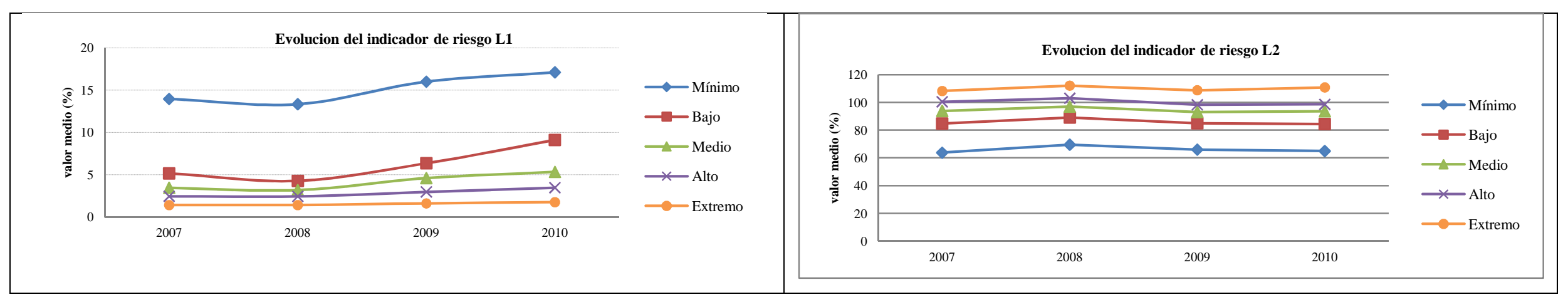

REVESCO No 109 - MONOGRÁFICO: La financiación complementaria y la respuesta de la economía social: la situación del "des-crédito" bajo la crisis financiera -

ISSN: 1885-8031 - www.ucm.es/info/revesco 